\title{
A THEORETICAL STUDY OF A MEMBRANE/ENZYME REACTOR WITH SINUSOIDAL ULTRAFILTRATION SWING
}

\author{
IN Ho KIM AND Ho NAM CHANG \\ Department of Chemical Engineering, Korea Advanced Institute of Science \\ and Technology, Cheongyangri, P. O. Box 150, Seoul, Korea
}

\begin{abstract}
A general model for the sinusoidal convective operation of a two-compartment membrane enzyme reactor was suggested to investigate theoretically the effect of ultrafiltration on reactor performance. From a phenomenological theory for membrane and solvent balance in the two regions separated by an ultrafiltration membrane, a pair of governing equations and physically meaningful dimensionless variables were obtained. Damköhler number $\left(\lambda^{2}\right)$, ratio of convective to diffusive fluxes $(F)$ and dimensionless frequency $(W)$ well explained the behavior of the reactor in batch mode, and residence time parameter $\left(L^{-1}\right)$ was added to study the performance in continuous mode.

In the batch reactor, the data from numerical simulation were fitted to the exponential decay proposed by Furusaki et al. and decay slope was studied in view of $\lambda^{2}, F$ and $W$. Conversion enhancement in the continuous reactor was observed in a diffusion-controlled region. Dimensionless frequency $W$ was related to the volume change in the two compartments. It was found that lower frequency favors conversion under the restriction of $W>F$.
\end{abstract}

\section{Introduction}

The immobilization of enzymes using semipermeable membrane has attracted the interest of many bioengineers in several aspects. Physical entrapment of enzyme in membrane devices such as ultrafiltration cells or hollow-fiber modules allows easier handling of enzyme reaction. ${ }^{10)}$ The membrane acts as a barrier for separating enzyme from substrate when the mixture of reactant and product is ultrafiltered after reaction ${ }^{6}{ }^{6}$ or segregated from substrate solution during reaction. ${ }^{7)}$ Thus in the latter case substrate molecules should pass the membrane phase to reach catalytic sites on the enzyme. There are two types of transfer mechanism for contact of the substrate with the enzyme: diffusive and convective transport. Waterland $e t$ al $^{8)}$ have considered the diffusive flux across the sponge region of hollow fiber theoretically, and confirmed their model by experiments. ${ }^{9)}$ Their paper commented on ultrafiltration effect on the total substrate flux in view of enzyme washout. On the other hand, convective transport plays a major role in the study of Alfani et al. ${ }^{11}$ They made a gel layer of enzyme and polymer on the ultrafiltration membrane through which substrate passes and reacts with enzyme in the gel layer.

Furusaki et al..$^{21}$ proposed a new reactor resembling a diffusion cell, and pressurized the compartment containing enzyme solution and that for reactant solution alternately, which leads to forward and backward convective transport. They performed experiments with catalase and hydrogen peroxide and fitted their experimental data to a simple theory of

Received January 18, 1982. Correspondence concerning this article should be addressed to $\mathrm{H}$. N. Chang. their own. However, the assumption in the derivation of the theory is so qualitative that the theory cannot be used to study the phenomena occurring in the reactor. In a recent paper, Furusaki and Miyauchi ${ }^{3}$ considered the diffusion contribution to reactant transport by solving a convective-diffusion equation with boundary conditions analogous to those of the penetration theory. The analysis showed how the concentration on the membrane surface was renewed and transport rate increased.

It is the purpose of the present investigation to propose a macroscopic model containing convective and diffusive fluxes for the reactor developed by Furusaki et al. and to show that convective transport helps the substrate to meet the enzyme in the diffusion-controlled region. The model equations are made into dimensionless forms from which a number of dimensionless parameters having physical significance can be extracted. In addition, it can be shown that it is possible theoretically to induced alternating convective transport by flow oscillation. The oscillatory flow is introduced into the reactor by a pulsatile feed stream, and the difference between the pulsatile feed and the steady outflow is applied to the membrane barrier.

\section{Theoretical Formulation}

\subsection{Pressure swing model}

As proposed by Furusaki et al., a modified version of their reactor is shown schematically in Fig. 1. Using solenoid valves with timer, we apply pressure to the two compartments separated by a membrane. The enzyme contained in the left side cannot pass the membrane, while the reactant solute and water in the right side are allowed to enter the enzyme side. The 


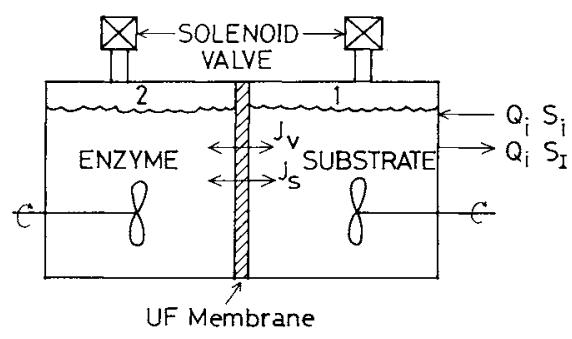

Fig. 1. Schematic diagram of model membrane/enzyme reactor with pressure swing.

reactor may be operated in continuous mode by feeding substrate solution into the right side and withdrawing the solution at the same rate as the feed.

To set a material balance around the two compartments, the phenomenological transport equation used by Kunitomo et al. ${ }^{5)}$ will be used:

$$
J_{s}=P_{m} \Delta C+s J_{v} C_{r}
$$

where $C_{r}=$ retained solute concentration, $J_{s}=$ solute flux, $J_{v}=$ solvent flux, $P_{m}=$ membrane permeability, $\Delta C=$ concentration difference between the two compartments, and $s=$ sieving coefficient $\left(C_{f} / C_{r}\right)$.

The sieving coefficient is the ratio of filtrate solute concentration to retained solute concentration. This value becomes $1-\sigma$ in the asymptotic range of $J_{v}$, where $\sigma$ is the Staverman reflection coefficient. ${ }^{4)}$

If we assume that concentration polarization is small enough to be neglected and $J_{v}$ varies sinusoidally without time lag, the resulting mass balances are:

$$
\begin{gathered}
V_{\mathrm{I}}=V_{\mathrm{IO}}-\frac{P_{a} A}{\omega}(1-\cos (\omega t)) \\
V_{\mathrm{II}}=V_{\mathrm{II} 0}+\frac{P_{a} A}{\omega}(1-\cos (\omega t)) \\
\frac{d\left(V_{\mathrm{I}} S_{\mathrm{I}}\right)}{d t}=-A J_{s}+Q\left(S_{i}-S_{\mathrm{I}}\right) \\
\frac{d\left(V_{\mathrm{II}} S_{\mathrm{II}}\right)}{d t}=A J_{s}-V_{\mathrm{II}} R\left(S_{\mathrm{II}}\right)
\end{gathered}
$$

where

$$
\begin{gathered}
J_{s}=P_{m}\left(S_{\mathrm{I}}-S_{\mathrm{II}}\right)+s J_{v} f\left(S_{\mathrm{I}}, S_{\mathrm{II}}\right) \\
J_{v}=P_{a} \sin (\omega t) \\
f\left(S_{\mathrm{I}}, S_{\mathrm{II}}\right)= \begin{cases}S_{\mathrm{I}} & \text { during forward cycle } \\
S_{\mathrm{II}} & \text { during backward cycle }\end{cases} \\
R\left(S_{\mathrm{II}}\right)=\frac{V_{\mathrm{max}} S_{\mathrm{II}}}{K_{m}+S_{\mathrm{II}}}
\end{gathered}
$$

Equations (2) and (3) represent the solvent balance, while Eqs. (4) and (5) stand for the solute balance. The solute flux in Eq. (6) is the outcome from Eq. (1) and Eq. (7) means the arbitrary sinusoidal variation of solvent flux. The kinetic equation for the reaction

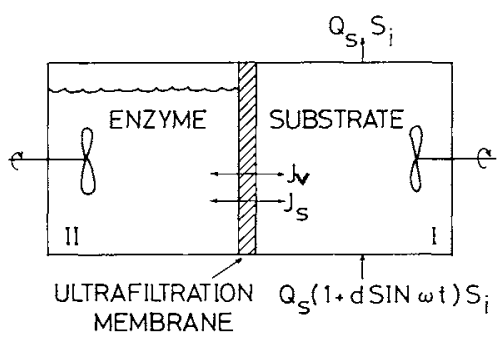

Fig. 2. Pulsatile flow model for inducing cyclic ultrafiltration.

term in Eq. (5) is given by a typical MichaelisMenten form. When Eqs. (4) and (5) are formulated, we assume that the compartments are in a wellmixed state.

For further development, Eqs. (2)-(9) are transformed by introducing dimensionless variables. The pertinent dimensionless variables are:

$$
\begin{aligned}
W & =\omega V_{\mathrm{IO}} / P_{m} A, \text { dimensionless frequency } \\
F & =P_{a} / P_{m}, \text { ratio of convection to diffusion } \\
\tau & =\omega t, \text { dimensionless time } \\
L & =Q / P_{m} A \text {, dimensionless flow rate } \\
S_{1} & =S_{\mathrm{l}} / S_{i}, \text { normalized concentration } \\
\widetilde{K}_{m} & =K_{m} / S_{i}, \text { normalized Michaelis constant } \\
\gamma & =V_{\mathrm{IIO}} / V_{\mathrm{I} 0}, \text { volume ratio } \\
\lambda^{2} & =V_{\max } V_{\mathrm{II}} / P_{m} A S_{i}, \text { Damköhler number }
\end{aligned}
$$

The final dimensionless equations for substrate mass balance have the following form:

$$
\begin{aligned}
V_{1} \frac{d S_{1}}{d \tau}-F S_{1} \sin \tau= & -\left(S_{1}-S_{2}\right)-s F f\left(S_{1}, S_{2}\right) \sin \tau \\
& +L\left(1-S_{1}\right) \\
V_{2} \frac{d S_{2}}{d \tau}+F S_{2} \sin \tau= & \left(S_{1}-S_{2}\right)+s F f\left(S_{1}, S_{2}\right) \sin \tau \\
& -\frac{\lambda^{2} S_{2}}{\tilde{K}_{m}+S_{2}}
\end{aligned}
$$

where

$$
\begin{aligned}
& V_{1}=W-F(1-\cos \tau) \\
& V_{2}=\gamma W+F(1-\cos \tau)
\end{aligned}
$$

\subsection{Pulsatile flow model}

Oscillatory motion of water can be generated by another method. To explain this method, we refer to Fig. 2. When the feed stream varies sinusoidally in flow rate and the efflux is maintained at the mean value of the inlet flow, the fluctuating component should come into and out of the enzyme compartment. In this instance we set up the substrate mass balance to examine the possibility of filtrate swing:

$$
\begin{aligned}
V_{\mathrm{IO}} \frac{d S_{\mathrm{I}}}{d t}= & -P_{m} A\left(S_{\mathrm{I}}-S_{\mathrm{II}}\right)-Q_{s} d s f\left(S_{\mathrm{I}}, S_{\mathrm{II}}\right) \sin (\omega t) \\
& +Q_{s}(1+d \sin (\omega t)) S_{i}-Q_{s} S_{\mathrm{I}}
\end{aligned}
$$




$$
\begin{aligned}
V_{\mathrm{II}} \frac{d S_{\mathrm{II}}}{d t} & +Q_{s} d S_{\mathrm{II}} \sin (\omega t)=P_{m} A\left(S_{\mathrm{I}}-S_{\mathrm{II}}\right) \\
& +Q_{s} d s f\left(S_{\mathrm{I}}, S_{\mathrm{II}}\right) \sin (\omega t)-\frac{V_{\mathrm{II}} V_{\max } S_{\mathrm{II}}}{K_{m}+S_{\mathrm{II}}}
\end{aligned}
$$

After transforming Eqs. (13) and (14) into dimensionless forms with the variables given in Eq. (10), we obtain the following dimensionless equations:

$$
\begin{gathered}
W \frac{d S_{1}}{d \tau}=-\left(S_{1}-S_{2}\right)-L_{s} d s f\left(S_{1}, S_{2}\right) \sin \tau \\
+L_{s}(1+d \sin \tau)-L_{s} S_{1} \\
V_{2}^{\prime} \frac{d S_{2}}{d \tau}+L_{s} d S_{2} \sin \tau= \\
\left(S_{1}-S_{2}\right)+L_{s} d s f\left(S_{1}, S_{2}\right) \sin \tau \\
-\frac{\lambda^{2} S_{2}}{\tilde{K}_{m}+S_{2}}
\end{gathered}
$$

where the new variables are defined as $L_{s}=Q_{s} / P_{m} A$ and $V_{2}^{\prime}=\gamma W+L_{s} d(1-\cos \tau)$. When there is no appreciable volume change in the compartments, pressure swing and pulsatile flow models will have the same performance. Comparing Eqs. (11) and (12) with Eqs. (15) and (16), we see that when $L_{s} d$ equals $F$, the latter pair of equations reduces to the former. Therefore, the cyclic operation resulting from pressure swing leads to the same performance of the flow pulsation.

\section{Numerical Simulation and Discussion}

The governing Eqs. (11) and (12) are simulated by varying the parameters $L, W, F$ and $\lambda^{2}$. In the following analysis $\widetilde{K}_{m}$ was set to 1 .

\subsection{Batch operation}

The parameter $L$ standing for feed flow rate was set to zero so that the reactor is operated in batch mode. Figure 3 shows that substrate concentration declines in a cyclic manner. To compare the case with different values of parameter, a simplified form of performance equation is derived as follows.

If the parameter $F$ is large enough to neglect the contribution by diffusion and the solute is small enough to assume that $s=1$, Eqs. (11) and (12) can be expressed by:

$$
\begin{aligned}
& V_{1} \frac{d S_{1}}{d \tau}=F\left(S_{1}-f\left(S_{1}, S_{2}\right)\right) \sin \tau \\
& V_{2} \frac{d S_{2}}{d \tau}=F\left(-S_{2}+f\left(S_{1}, S_{2}\right)\right) \sin \tau-\frac{\lambda^{2} S_{2}}{1+S_{2}}
\end{aligned}
$$

Further simplification can be made when $\lambda^{2} \gg F$ and $W \gg F$. The substrate concentration in the enzyme side nearly equals zero in the former case and there is no volume change in the latter case. When we assume these conditions, Eq. (17) reduces to

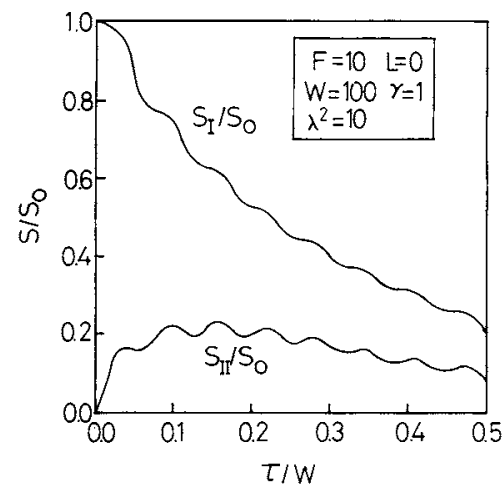

Fig. 3. Batch operation example: cyclic decay of reactant concentration.

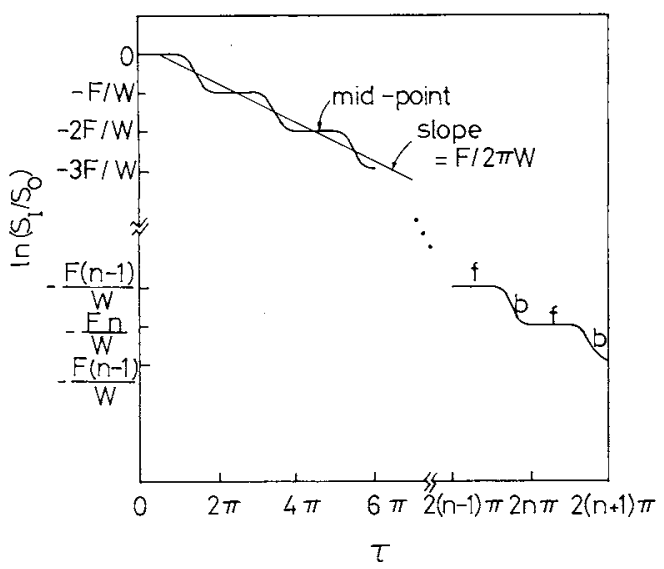

Fig. 4. Calculation of decay slope for sine-wave pressure swing.

$W \frac{d S_{1}}{d \tau}= \begin{cases}0 & \text { during forward cycle } \\ F S_{1} \sin \tau & \text { during backward cycle }\end{cases}$

After integration of Eq. (19) the final form of the performance equation appears to be:

$S_{1}=S_{0 n}$ during forward cycle

$S_{\mathrm{I}}=S_{0 n} \exp \left(-\frac{F}{W} \cos \tau_{n}\right)$ during backward cycle

where $S_{0 n}$ represents the intial value of $S_{\mathrm{I}}$ in the $n$-th cycle and $\tau_{n}$ ranges from $\pi$ to $2 \pi$. Equation (20) is explained by Fig. 4. Connecting the mid-points in the forward cycle, we calculate the decay slope, which is $F / 2 \pi W$. For the square wave used by Furusaki et $a l .{ }^{2)}$ the slope is $F / 2 W$. Hence the sinusoidal wave is not so effective as the square wave by the factor of $1 / \pi$.

For the present sinusoidal pressure swing, an arbitrary coefficient $\alpha$ is introduced to fit the numerical simulation data with it:

$$
S_{\mathrm{I}}=S_{0} \exp \left(-\frac{\alpha F}{2} \frac{\tau}{W}\right)
$$

Representative data obtained from the numerical simulation of Eqs. (11) and (12) are plotted as 


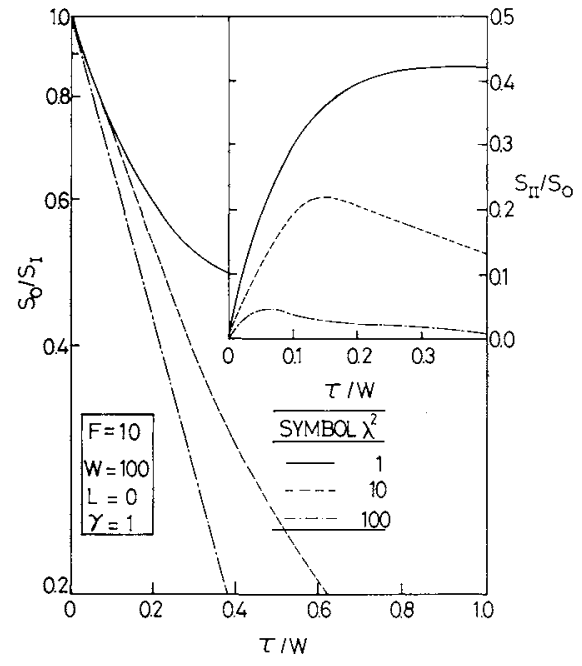

Fig. 5. Dependency of concentration decay on $\lambda^{2}$.

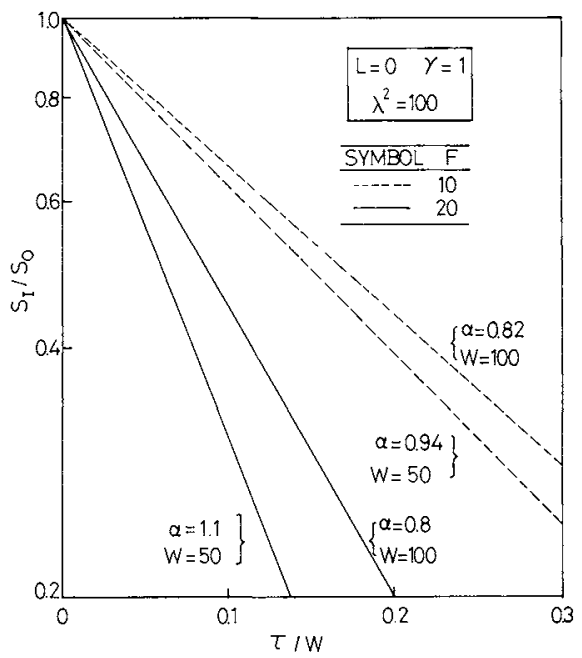

Fig. 6. Dependency of concentration decay on $F$ and $W$.

$\ln \left(S_{\mathrm{I}} / S_{0}\right)$ vs. $\tau / W$ by connecting inflection points of the fluctuating wave. Figures 5 and $\mathbf{6}$ show that concentration decay depends on $F, W$ and $\lambda^{2}$. The effect of $\lambda^{2}$ is shown in Fig. 5. Substrate concentration decays much faster in the case of high Damköhler number $\lambda^{2}$, which is the measure of reaction rate over diffusion rate. The exponential decay of the substrate compartment concentration is well illustrated at $\lambda^{2}=$ 100 when the enzyme compartment concentration nearly equals zero. Meanwhile, the decay at $\lambda^{2}=1$ does not follow the exponential decline curve due to high concentration in the enzyme compartment.

The effect of dimensionless frequency $W$ is also examined in Fig. 6. The decay slope is steeper as $W$ decreases and $F$ increases. Lower frequency results in higher volume change in each compartment and substrate reacts with enzyme more easily. This observation was also reported by Furusaki et al. ${ }^{2)}$ We can see in Fig. 6 that cycle time has less effect on performance at a low $F$. This indicates that as $F$

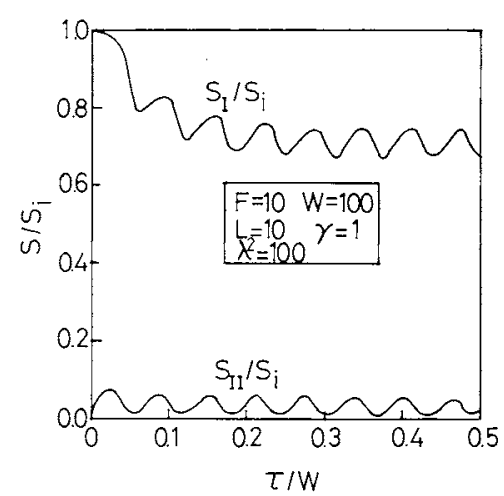

Fig. 7. Continuous operation example: sustained oscillation of reactant concentration.

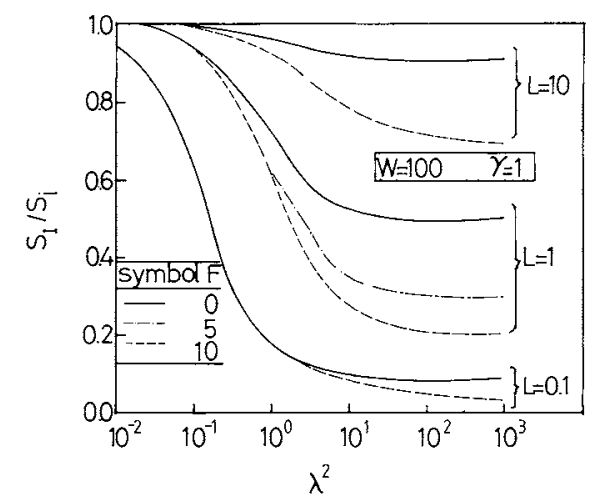

Fig. 8. Efflux concentration versus Damköhler number at different $F$ and $L$.

decreases, diffusive flux masks convective flux. The coefficient $\alpha$ covers the deviation from the assumption of $\lambda^{2} \gg F$ and the difference between the square and sine waves. The values of $\alpha$ are in the range of near one, which implies that the simple model of Furusaki et al. $^{2)}$ well describes the pressure swing batch reactor. The inefficiency in using sine wave is not so large as expected because diffusion occurs during pressure swing operation.

\subsection{Continuous operation}

The performance of the membrane/enzyme reactor in continuous mode is simulated by varying $\lambda^{2}$ with $F$ and $L$ as parameters. For illustration, a concentration oscillation is shown in Fig. 7. Substrate solution moves into the enzyme side during the forward cycle and substrate concentration increases in both compartments; concentration declines during the backward cycle due to depletion in the enzyme side and dilution in the substrate side. By calculating mean values of the wave, we obtain the conversion.

Figure 8 shows the outlet mean concentration vs. Damköhler number. The parameter $L$ represents the reciprocal of residence time. It is varied from 0.1 to 10 to observe the conversion change according to flow rate increase. As the residence time becomes longer, the transition from the kinetic-controlled region to 
the diffusion-controlled region occurs at smaller value of $\lambda^{2}$. In the diffusion-controlled region the conversion at $F=0$ approaches an asymptotic line where no further decrease in concentration occurs. The asymptotic value of conversion is given by:

$$
S_{1}=1-(1+L)^{-1}
$$

Equation (22) stems from the performance equations that calculate the conversion without pressure swing. The equations can be derived from Eqs. (11) and (12) with $F=0$ and $d S / d \tau=0$.

$$
\begin{aligned}
1-S_{1} & =(1+L)^{-1}\left(1-S_{2}\right) \\
S_{2} & =\frac{1}{2}\left(a+\sqrt{a^{2}+4 \tilde{K}_{m}}\right)
\end{aligned}
$$

where

$$
a=1-\widetilde{K}_{m}-\lambda^{2}\left(1+L^{-1}\right)
$$

With Eqs. (23) and (24) minimum conversion owing to diffusion is calculated and plotted as solid lines in Fig. 8. The effect of $F$ is also shown through the conversion increase with $F$ going up. The contribution of ultrafiltration to substrate transport is clearly seen in the diffusion-controlled region. As another extreme case the kinetic-controlled asymptote is $S_{\mathrm{I}} / S_{i}=1$, where conversion is negligible.

\section{Conclusion}

The present study provides a fundamental view of the cyclic operation in a membrane/enzyme reactor such as hollow fiber enzyme reactor and ultrafiltration cell reactor by lumping the system into two compartments and using a phenomenological law of transport in the membrane. Parameteric study of governing equations gives some notion about order of magnitude of parameters to make pressure swing effective for enhancing reactor performance. In addition, pulsed flow into enzyme reactor gives rise to the same consequences as the pressure swing theoretically, and the operation may be used for improving enzyme reactor performance:

\section{Nomenclature}

$\begin{array}{ll}A & =\text { mass transfer area } \\ C & =\text { concentration } \\ C_{f} & =\text { filtrate solute concentration } \\ C_{r} & =\text { retained solute concentration } \\ d & =\text { fraction of mean flow rate } Q_{s} \\ F & =\text { ratio of convection to diffusion } \\ f\left(S_{1}, S_{2}\right) & =\text { function defined by Eq. }(8)\end{array}$

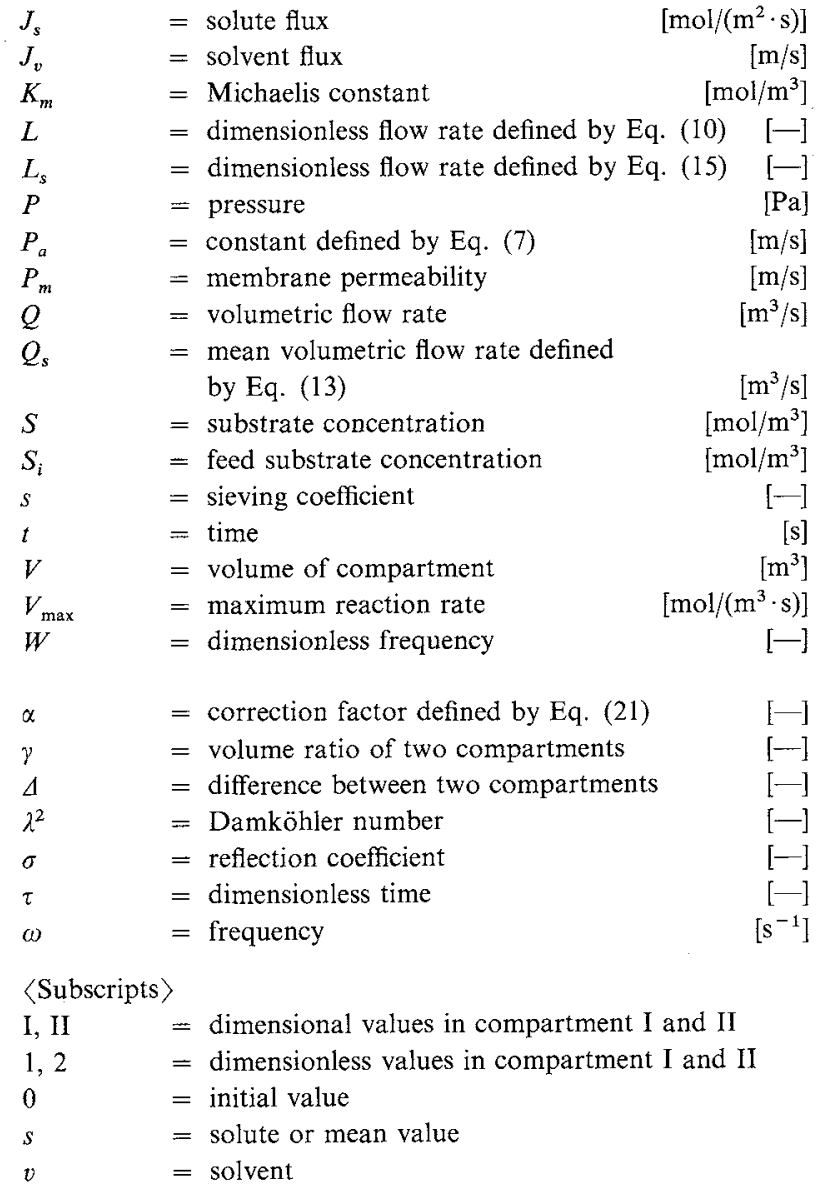

\section{Literature Cited}

1) Alfani, F., G. Iorio, G. Greco, Jr., M. Cantarella, M. H. Remy and V. Scardi: Chem. Eng. Sci., 34, 1213 (1979).

2) Furusaki, S., T. Kojima and T. Miyauchi: J. Chem. Eng. Japan, 10, 233 (1977).

3) Furusaki, S. and T. Miyauchi: J. Chem. Eng. Japan, 14, 479 (1981).

4) Kedem, O. and A. Katchalsky: Biochim. Biophys. Acta, 27, 229 (1958).

5) Kunitomo, T., E. G. Lowri, S. Kumazawa, M. O'Brien, J. M. Lazarus, M. N. Gottlieb and J. P. Merrill: Trans. ASAIO, 23, 234 (1977)

6) O'Neill, S. P., P. Dunnill and M. D. Lilly: Biotech. Bioeng., 13, 320 (1971).

7) Rony, P. R.: Biotech. Bioeng., 13, 431 (1971).

8) Waterland, L. R., A. S. Michaels and C. R. Robertson: AIChE J., 20, 50 (1974).

9) Waterland, L. R., C. R. Robertson and A. S. Michaels: Chem. Eng. Commun., 2, 37 (1975).

10) Zaborsky, O. S.: "Immobilized Enzymes," p. 103, CRC Press, Cleveland (1973). 